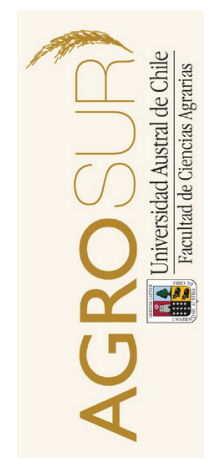

\title{
Antecedentes de control de Hieracium pilosella L. para su aplicación en la Región de Magallanes y Antártica Chilena: Una revisión
}

\author{
Background of Hieracium pilosella L. control for their application in the Chilean \\ Magallanes and Antarctic Region
}

Ivelic-Sáez, J. ${ }^{a *}$, Radic, S. ${ }^{b}$ Domínguez, E. ${ }^{a}$, Salinas, C. ${ }^{b}$

${ }^{a}$ Instituto de Investigaciones Agropecuarias, Centro Regional Kampenaike. Angamos 1056, Punta Arenas, Chile

${ }^{b}$ Departamento de Ciencias Agropecuarias y Acuícolas, Universidad de Magallanes, Avenida Bulnes 01855 , Punta Arenas, Chile.

\begin{tabular}{l} 
A R T I C L E I N F O \\
\hline Article history: \\
Received 04.08.2017 \\
Accepted 14.11.2017 \\
\hline Keywords: \\
Hieracium pilosella L. \\
Weeds control \\
Grasslands degradation \\
Patagonia \\
\hline Original Research Article, \\
Animal Science \\
\hline *Corresponding author: \\
Jorge Ivelic-Sáez \\
E-mail address: \\
jorge.ivelic@inia.cl
\end{tabular}

\author{
A B S T R A C T
}

Hieracium pilosella is classified as a noxious weed at the Chilean Patagonia. Its explosive expansion represents an economic loss for livestock production because its great competitive ability impedes growth of more productive species. Its expansion is due to soils degradation caused by overgrazing. There are different types of weed control such as chemical, mechanical and biological, but H. pilosella control and eradication require proper animal management to prevent soil degradation and an integrated preventive management to avoid the spread of the plant.

\section{RESUMEN}

Hieracium pilosella es una planta catalogada como maleza en la región de Magallanes la cual debido a su explosiva expansión representa una pérdida económica en el rubro ganadero debido a la gran habilidad competitiva, lo que evita el crecimiento de especies de mayor calidad forrajera. Su rápida expansión se debe a la degradación de los suelos que presentan algunos sectores de la región debido al sobrepastoreo entre otros factores. Existen varios tipos de controles tales como químicos, mecánicos y biológicos, sin embargo, para el control y erradicación de esta maleza se debe tener un correcto manejo animal para evitar la degradación de los suelos y un manejo preventivo e integral para evitar la diseminación de la planta.

Palabras clave: Hieracium pilosella L., control de malezas, degradación de praderas, Patagonia.

\section{INTRODUCCIÓN}

Según el Censo Agropecuario, la Región de Magallanes posee cerca de 13 millones de hectáreas, de las cuales 5.357.442 tienen relación con predios de uso agropecuario (INE, 2007), los que poseen una superficie promedio aproximada de 4.000 hectáreas. Con una producción ganadera principalmente ovina, basada en su mayoría en sistemas de producción extensivos con pastoreo de praderas naturales. El SAG (2003, 2004a, 2004 b) realizó un estudio de la vegetación que abarcó la superficie de uso agropecuario regional, donde se determinaron diferentes tipos de comunidades vegetales, algunas de las cuales han sido afectadas por una maleza de rápido crecimiento conocida como Hieracium pilosella (Linneo).

En género Hieracium es un grupo de especies herbáceas del hemisferio norte perteneciente a la familia de las Asteraceas (Pisano, 1977). En la región de Magallanes están presentes varias especies de Hieracium descritas por Moore (1993) tales como; Hieracium murorum L., Hieracium antarcticum D’Urv, Hieracium patagonicum Hooker, cuya principal característica es la ausencia de estolones. Por otro lado Hieracium pilosella L. y Hieracium flagellare Willd, presentan estructuras vegetativas como estolones o rosetas (Moore, 1983).

$H$. pillosella resulta relevante en la ganadería magallánica por invadir rápidamente los pastizales natu- 
rales de la región, afectando la biodiversidad y disminuyendo la producción pratense (Domínguez, 2012). Además, esta planta es considerada cuarentenaria por los países del MERCOSUR, con los cuales Chile mantiene relaciones comerciales (SAG, 1998).

Debido al fuerte impacto económico que tiene en la región de Magallanes, es que se realiza una revisión de las diferentes estrategias de control y/o erradicación de H. pilosella.

\section{Características de H. pilosella}

La Pilosella u Oreja de Ratón (en inglés Mouseear o Hawkweed) posee una gran capacidad competitiva, debido a la producción de tallos rastreros o estolones desde una roseta basal (Covacevich et al., 1995). Los mismos autores mencionan que desde la detección de esta hierba a partir de los años 80 ', ha presentado un aumento gradual, encontrándose en una fase de expansión acelerada. Hoy en día esta planta se encuentra propagada por toda la región de Magallanes, tanto en el continente como en la isla Tierra del Fuego (Domínguez, 2012).
Según Strauch (2012), H. pilosella está diseminada por ambas vertientes del Estrecho de Magallanes siendo favorecida su expansión hacia el interior por la degradación progresiva de los suelos de la región, ya que esta planta coloniza el suelo desnudo y prolifera por la poca competencia del pastizal presente en suelos degradados.

Por otro lado, von Moltke (2009), menciona que el aumento de las poblaciones de $\mathrm{H}$. pilosella son considerables desde 1994 hasta 2009, ya que en aquellos años solo se encontraba en el sector sur de Punta Arenas en la zona de la Discordia. En el 2004 se observan poblaciones en el parque nacional Pali-Aike y en toda la provincia de Magallanes incluyendo Isla Riesco, sin embargo, para esa fecha todavía no había vestigios de esta hierba en Morro Chico, Rio Pérez y Teniente Merino, lugares en donde hoy en día si se encuentra. En la actualidad esta planta se puede hallar asociadas a formaciones vegetales de praderas húmedas y coirón.

H. pilosella posee estolones hojosos (Figura 1). Hojas de 8 a $12 \mathrm{~cm}$ de largo por 0,5 a $2 \mathrm{~cm}$ de ancho, las bases en roseta, oblanceoladas, con pelos ralos, simple y largos en ambas caras; cara inferior cubierta densa-

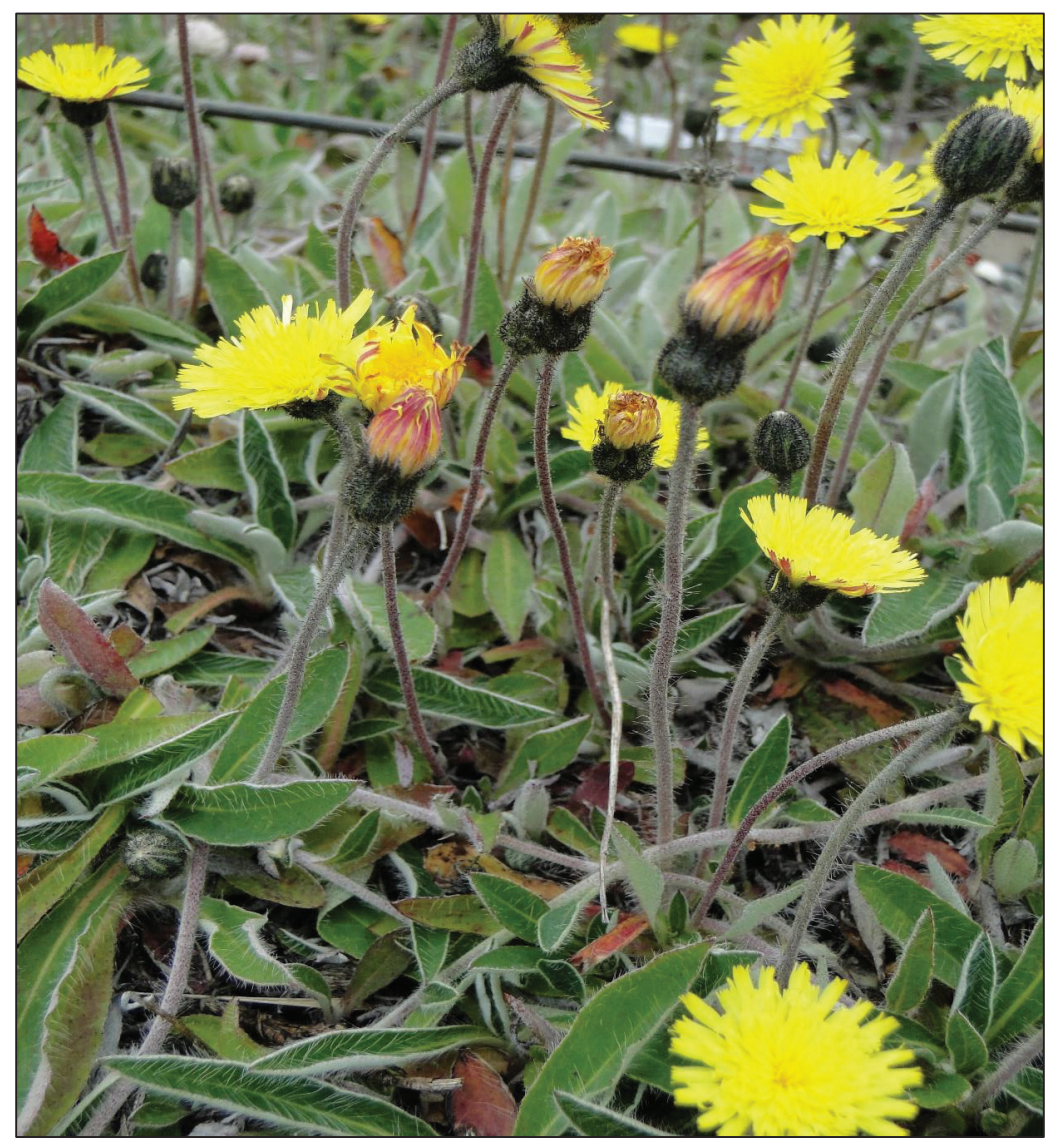

Figura 1. Planta de H. pilosella.

Figure 1. H. pilosella plant. 
mente de pelos estrellados. Escapo floral con solo un capítulo hasta $30 \mathrm{~cm}$ de alto, cubierto de pelos simples y estrellados. Brácteas involúcrales de 8 a $12 \mathrm{~mm}$ de largo por 0,7 a $1,5 \mathrm{~mm}$ de ancho, linear, lanceoladas, agudas, con pelos simples y glandulosos estrellados, negros. Flores todas liguladas, lígula amarilla con una franja roja en el dorso. Aquenios de 2,2 a 2,5 mm de largo por $0,5 \mathrm{~mm}$ de ancho, estriados, obcónicos, crenados en el ápice. Pappus de 4,5 a 5,5 mm con pelos simples (Domínguez, 2012).

En Magallanes, la maleza inicia su crecimiento a mediados de agosto con la aparición de hojas verde claro en el centro de la roseta formada por hojas verde rojizas que han pasado el invierno. La aparición de la inflorescencia y estolones se produce e noviembre, para lo cual es necesario que primero haya inicio de escapo floral antes del esbozo de los estolones (SAG, 1998). El capítulo o flor se desarrolla completamente antes que el tallo se elongue a partir de la roseta, siendo dicho alargamiento muy rápido (SAG, 1998). La semilla madura durante febrero y es dispersada normalmente por viento. Los estolones son delgados y crecen cerca de la vegetación nativa siendo rara vez consumidos por los animales, logrando anclarse en el suelo en toda su extensión por medio de raíces producidas en los nudos (SAG, 1998). En el extremo terminal de cada estolón aparece una nueva planta, esta progenie se enraíza en el suelo después de las primeras lluvias cuando se ha detenido el crecimiento del estolón. Las plantas hijas ocasionalmente ocupan espacio formando una masa con la planta madre, aunque lo más común es que se establezcan más alejadas, extendiendo la colonia. En invierno las hojas exteriores de los rosetones se ponen rojizas y se mueren (Bishop y Davy, 1994). H. pilosella depende casi exclusivamente de su reproducción vegetativa para el mantenimiento y la expansión de la población (Bishop y Davy, 1994; Makepeace 1985a). Produciéndose rosetas hijas estacionalmente desde los estolones o de las hojas axilares de los rosetones senescentes (SAG, 1998).

\section{Características del medio ambiente circundante}

Bishop y Davy (1994), señalan que H. pilosella posee intolerancia a altas temperaturas. Se desarrollan en áreas pluviometrías relativamente bajas (475-800 mm) en Nueva Zelanda (Makepeace, 1985b), sin embargo, en la Patagonia Chilena se encuentra distribuida desde el sector pampeano hasta la zona húmeda de la región (Figura 2) presentándose en un gradiente de precipitación de 200 hasta 500 mm (Cruz y Lara, 1987).

H. pilosella se encuentra en una amplia gama de suelos, aunque es típica de aquellos que tienen un bajo estado nutritivo, con bajos contenidos de nitrógeno y fósforo, característicos de suelos sobrepastoreados (Bishop y Davy, 1994). Además, H. pilosella se confina a suelos relativamente bien drenados y al parecer no tolera agua cerca de la superficie. El sistema radical es generalmente superficial, mientras que estudios de excavación sugieren que normalmente se extiende a una profundidad de sólo 10-15 cm (Anderson, 1927; Bornkamm, 1958).

Bishop y Davy (1994), registraron que H. pilosella está casi totalmente restringida en suelos de $\mathrm{pH}$ mayor que 4,5 en la región de Sheffield Inglaterra, pero se informó alta frecuencia en praderas degradadas sobre suelos de pH entre 3,7 y 4,8 en Nueva Escocia, Canadá.

Boswell y Espie (1998), estudiaron los efectos de $H$. pilosella sobre el suelo confirmando que esta planta aumenta la acidez del suelo y el contenido de aluminio soluble. Los efectos combinados de humedad reducida, baja disponibilidad de cationes, alto nivel de aluminio soluble e incremento de acidez del suelo, hacen que el ambiente circundante a $\mathrm{H}$. pilosella sea desfavorable para el desarrollo de especies competitivas Boswell y Espie (1998).

El suelo bajo $H$. pilosella es más ácido que el suelo que rodea el manchón en 0,5 unidades de $\mathrm{pH}$ y contiene más carbono orgánico. Los valores de $\mathrm{Ca}$, Mg y $\mathrm{K}$ intercambiable son más bajos en las orillas del manchón y más alta en la superficie cubierta por $H$. pilosella. Se ha demostrado que bajo $H$. pilosella existe una mayor tasa de descomposición de materia orgánica haciéndola más lábil (McIntosh et al., 1995).

\section{Factores de influencia en la proliferación de H. pilosella}

Un gran número de autores (Estrada y Flory, 2015; Hernández et al., 2014; Mandal y Joshi, 2014) han establecido que la profileración exacerbada de $H$. pilosella se debe a la degradación de los suelos producto de factores antropogénicos como el sobrepastoreo y erosión. Rauber et al. (2016) indican que la capacidad de invasión de $H$. pilosella depende en mayor magnitud del tipo de perturbación al que haya sido sometido el suelo (desarrollo de caminos, líneas de gaseoductos, áreas quemadas y sobrepastoreo) y a la asociación vegetal circundante. Sin perjuicio de lo anterior, la proliferación de $H$. pilosella responde a una dinámica de poblaciones compleja en donde factores bióticos, abióticos, así como la escala espacial de la invasión influyen en el éxito de esta planta (Rauber et al., 2013).

En estudios realizados en la isla Tierra del Fuego Argentina queda demostrado que la invasión temprana está asociada a comunidades de Chiliotrichum diffusum Foster F. (mata negra o romerillo) y Poa spiciforme Steud., las cuales han estado bajo constante pastoreo ovino durante largos periodos. Esto, debido a que este tipo de comunidades vegetales están presente en suelos con mayor fertilidad y alto contenido de humedad. (Rauber et al., 2013). De estos estudios se desprende 


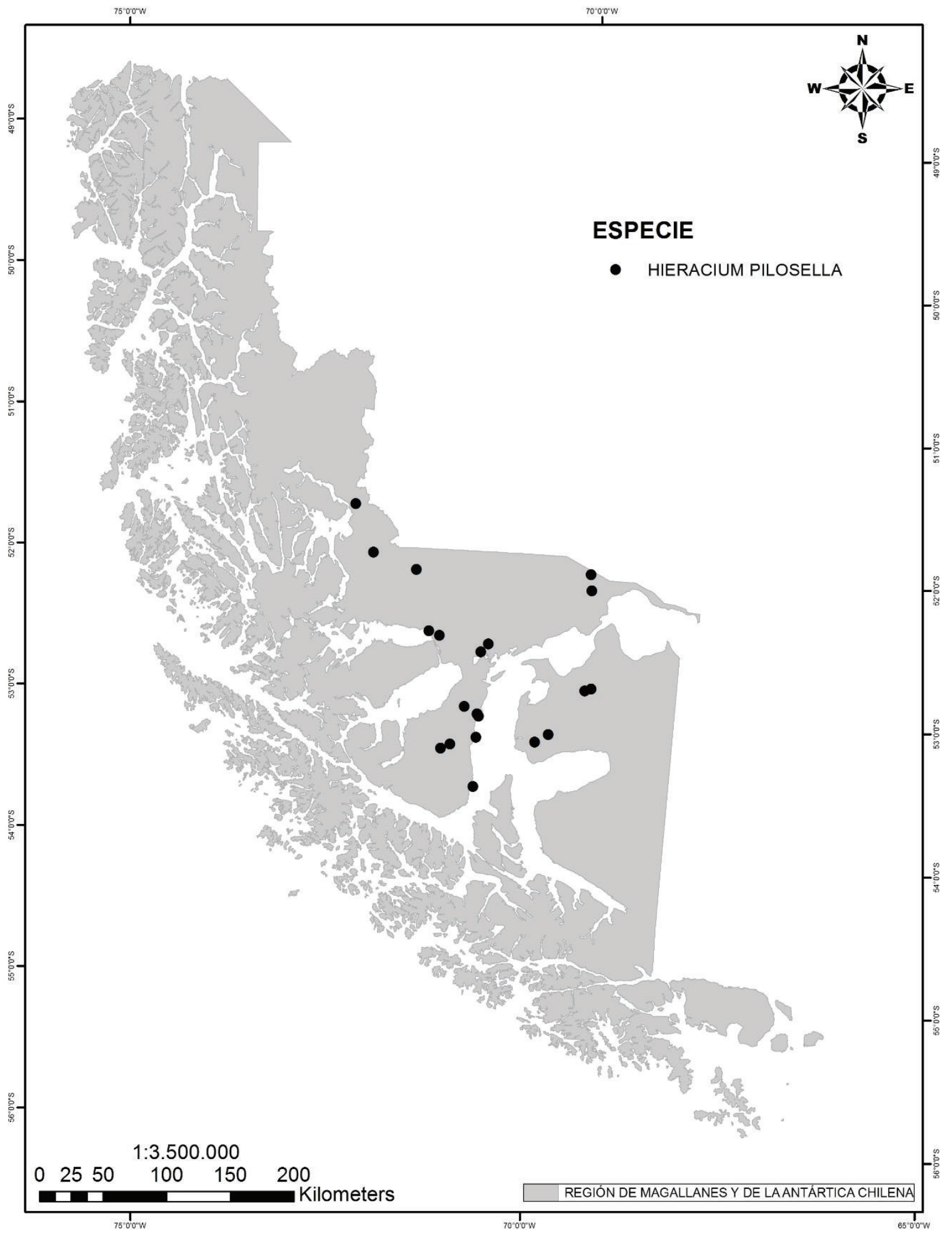

Figura 2. Distribución de Hieracium pilosella en la región de Magallanes. (Adaptado de Domínguez et al., 2011).

Figure 2. Hieracium pilosella distribution in Magallanes. (Adapted from Domínguez et al., 2011). 
que la proliferación de la planta invasora depende del nivel de perturbación o estreses mecánicos sobre la vegetación primaria. La invasión de $H$. pilosella se hace pronunciada cuando el disturbio sobre las comunidades de $C$. diffusum son de media magnitud (Rauber et al., 2014). En este ámbito, cuando estas asociaciones vegetales son densas (sin ser pastoreadas o removidas) la capacidad de invasión de H. pilosella se hace nula debido a la poca capacidad de competencia por luz y al balance de la humedad del suelo que favorece a la mata negra. Además, $C$. diffusum es capaz de detener el esparcimiento de las semillas de $H$. pilosella provocado por los vientos característicos de la Patagonia. Por otro lado, en sectores de mata muy poco densos, la mayor influencia de la luz provoca un desecamiento exacerbado del suelo evitando la proliferación de $H$. pilosella y en suelos con niveles freáticos elevados y salinos (Rauber et al., 2016).

Al contrario, $H$. pilosella tiene una menor probabilidad de proliferar en suelos ácidos e infértiles (Collantes et al., 1999)

Saggar et al. (1999) demuestran en estudios en laboratorio, los suelos bajo $H$. pilosella presentan un acelerado reciclaje de carbono y una menor mineralización de nitrógeno, esto último relacionado a una liberación de elementos orgánicos complejos como polifenoles y ligninas. En el mismo ámbito, Knicker et al. (2000) mencionan que la diferencia en la fracción de carbono entre los suelos bajo $H$. pilosella y la vegetación aledaña se debe a la cantidad y calidad de los residuos exudados por las plantas de $H$. pilosella los cuales contienen compuestos fenólicos que, además, influirían en el secuestro y mineralización del nitrógeno.

Por una parte, Díaz-Barradas et al. (2015) en la Patagonia Argentina, indican que $H$. pilosella es capaz de modificar el balance de nitrógeno en las plantas aledañas a los parches a medida que va colonizando gracias a sustancias alelopáticas. Estas sustancias obligan a plantas nativas próximas al parche de H. pilosella a absorber el nitrógeno del suelo en forma orgánica, lo que implica un mayor gasto energético por parte de las plantas nativas para suplir las necesidades de nitrógeno.

Por otro lado, en Nueva Zelanda, Steer y Norton (2013), indican que la expansión de H. pilosella está influenciada por la altitud sobre el nivel del mar. Ellos concluyen que este género puede colonizar desde los 1.200 hasta $1.800 \mathrm{~m} \mathrm{~s}$. n. m.

Finalmente, Beckmann et al. (2012), en una comparación de plantas de $H$. pilosella de Alemania y Nueva Zelanda, mencionan que la planta responde positivamente al aumento de la radiación UV-B (condición actual en el Hemisferio Sur), produciendo hojas más largas y mayor biomasa. Esto es comparable con lo que ocurre en la región de Magallanes la cual está expuesta directamente al adelgazamiento de la capa de ozono (Farman et al., 1985).

\section{DESARROLLO}

\section{Control y erradicación de H. pilosella.}

Por la naturaleza extensiva de los sistemas ganaderos en la región de Magallanes, los altos costos relativos de control y las limitaciones en las prácticas de manejo hacen que la prevención y control temprano sean la mejor estrategia (Strauch, 2012), sin embargo, va a depender del nivel tecnológico que posea el productor.

Rose y Frampton (1999) mencionan que el manejo del pastoreo es potencialmente una de las estrategias más factible para disminuir la expansión de $\mathrm{H}$. pilosella sobre terrenos de baja fertilidad. Covacevich y Cárdenas (2005), demuestran que el crecimiento y dispersión de $H$. pilosella es susceptible al pastoreo animal ya que modifica su hábito de crecimiento haciéndolo más postrado y con hojas más cortas. Además, indican que el pastoreo en épocas de floración aminora el avance de los manchones porque elimina los estolones más agresivos de los bordes y elimina o reduce la diseminación de las semillas. Por último, mencionan que las cargas altas disminuyen la selectividad por parte del animal asegurando el daño a las plantas aisladas de H. pilosella. Sin perjuicio de lo anterior, Covacevich y Cárdenas (2005) reportan que la calidad bromatológica de las hoja, flores y botones de $H$. pilosella poseen valores aceptables de energía metabolizable (2,86-2,90 Mcal g MS$^{-1}$ ), y de hecho el $74 \%$ de las hojas mostraron signos de consumo ovino o animales silvestres, mientras que la flor se aprecia visualmente que son apetecidas por el ganado.

Por otro lado, Espie (1992) señala que el pastoreo puede reducir la densidad y la abundancia de especies erectas, pero no controlará $H$. pilosella una vez que se ha establecido. Se recomiendan pastoreos poco intensivos durante la primavera y a inicio del verano para reducir la formación de semillas y limitar la abundancia de $H$. pilosella. La intensidad del pastoreo va relacionado a evitar la degradación de sectores que ya se encuentran en un estado de degeneración.

En el caso de control mediante pastoreo, la razón principal de rechazo animal es el hábito de crecimiento, con una roseta muy apegada al suelo, esto sumado a una baja palatabilidad que puede modificarse por efecto fertilizante; pero de todos modos el aporte de materia seca en el consumo es mínimo (Covacevich et al., 1995).

Bishop y Davy (1994), señalan que existen marcadas reducciones en población de $H$. pilosella en praderas que han sido mejoradas a través de tratamientos de fertilización en Canadá y en Nueva Zelanda. Asimismo H. pilosella está entre las primeras especies en ser suprimidas al existir pastoreo intensivo y quemas en la Isla de Rhum, Inglaterra (Bishop y Davy, 1994). Los mismos autores señalan que la densidad de rosetas 
tiende a disminuir luego de tres años de adiciones sucesivas de fertilización. La adición de nitrógeno y fósforo, con o sin potasio, causan una dramática reducción en densidad. La combinación de nutrientes adicionados tiende a reducir la densidad de plantas florecidas. El control también puede ser logrado mejorando el nivel de nutrientes sobre siembras de especies leguminosas forrajeras (Scott et al., 1990).

En general, la fertilización promueve la competencia de especies nativas y exóticas de mayor valor forrajero sobre todo en ausencia de pastoreo (exclusiones) como los demuestran Cipriotti et al. (2012).

En Nueva Zelanda, Cossens y Boswell (1993), han demostrado que la regeneración y la fertilización de praderas dominadas por $\mathrm{H}$. pilosella tienen un exitoso control sobre ella. También señalan que Trifolium hibridum L. fue el más efectivo competidor con H. pilosella. Strauch (2012), menciona que la fertilización fosforada y azufrada es un método complementario para el control de los manchones de esta maleza. Sin embargo, Makepeace (1985b), señala que la propagación vegetativa de $H$. pilosella se incrementa con la fertilización. El nitrato de calcio aumenta el número de plantas hijas producidas y el superfosfato fortificado con molibdeno aumenta el grosor de los estolones. Similares resultados arrojaron el estudio de Salinas (2002), dejando en claro que la mezcla de 2,4 D (720 $\mathrm{g} \mathrm{ha}^{-1}$ ) con fertilización nitrogenada (60 $\mathrm{U} \mathrm{ha}^{-1}$ ) aumenta la cantidad de estolones.

Aunque H. pilosella es característica de suelos deficientes de nitrógeno en Europa, experiencias en Canadá indican respuesta a la fertilización nitrogenada y fosforada en el aumento de su crecimiento (Makepeace, 1985b).

Estudios realizados en la provincia de Tierra del Fuego por Cipriotti et al. (2014) muestran resultados interesantes en esas latitudes. En dicho estudio se evaluaron diferentes medidas de control (siembra y fertilización, solo fertilización con fósforo y nitrógeno, aplicación de herbicidas selectivo y no selectivo) asociado a pastoreo y ausencia de este.

Los resultados demostraron que la fertilización en conjunto con la exclusión del pastoreo doméstico ovino permitieron exaltar la respuesta de la vegetación nativa y naturalizada del inter coirón, promoviendo un descenso significativo de la cobertura de H. pilosella, sobre todo por la gran competencia ejercida por Trifolium repens L. frente a H. pilosella. Según Cipriotti et al. (2014) la naturaleza de la respuesta interactiva observada puede obedecer en parte al estadío de la invasión, ya que durante las fases tempranas de esta invasión la cobertura de $H$. pilosella es baja y el espacio inter coirón está mayormente ocupado por un conjunto de especies deseables y diversas, con lo cual el pastoreo selectivo puede reducir la intensidad de competencia entre $\mathrm{H}$. pilosella y las especies palatables. En ese contexto, la fer- tilización y la exclusión del pastoreo, puede promover el crecimiento de competidores ya establecidos, resultando en un aumento rápido en la altura de la canopia que afecta la supervivencia de $H$. pilosella. Por tanto, en manchones muy grandes en donde $H$. pilosella es la especie con mayor cobertura, una fertilización solo promovería el crecimiento de $H$. pilosella. Los mismos autores mencionan la importancia del mantenimiento de la cobertura del suelo, indicando que en áreas muy invadidas y de gran extensión, cualquier práctica de remoción de H. pilosella debe ir acompañada de prácticas de recuperación de la cobertura vegetal para no dejar el suelo expuesto debido a que esta maleza aprovecha de manera eficiente el suelo libre de competencia para establecerse y dispersase mediante crecimiento vegetativo. Por lo tanto, el correcto manejo de los pastizales para evitar la degradación del suelo es fundamental. Manejos conservacionistas, disminución de carga animal y rezagos prolongados de potreros deben ser incluidos en la planificación de cualquier estancia ganadera.

H. pilosella es una maleza de hoja ancha que se controla prontamente con herbicidas. Se destruye de forma consistente por simples aplicaciones de MCPA, 2,4-D amina o éster, Mecoprop, o combinaciones de bajas dosis de 2,4-D con Mecoprop, Dichlorprop, Fenoprop o Dicamba (Bishop y Davy, 1994).

Makepeace (1985b), señala que la mezcla de 2,4-D Ester y Clopyralid aplicando $1000+400 \mathrm{~g} \mathrm{ha}^{-1}$ o 750 + $300 \mathrm{~g} \mathrm{ha}^{-1}$ fue el mejor control para H. pilosella. Esta aplicación causó daño al trébol (T. repens) presente en el periodo inmediato a la aplicación. Al año siguiente, el rebrote de $H$. pilosella fue más escaso en la parcela de mejor control, pero en parcelas pulverizadas con herbicidas menos efectivos, el rebrote fue similar a parcelas no pulverizadas. Estudios realizados en Estados Unidos (Clyde, 1992) muestran que el uso de 2,4-D tiene un buen control sobre especies de la misma familia; no así el uso de Dicamba del cual se obtiene de un pobre a un buen control. De esta manera, las combinaciones que mejoran el control en el experimento antes citado fueron: 2,4-D; 2,4-D + Triclorpyr; 2,4-D + Micoprop + Dicamba; MCPA + Mecorprop + Dicamba y Clopyralid + Triclopyr (Clyde, 1992).

Covacevich et al. (1995), realizaron ensayos en el cual utilizaron tres productos químicos: Clopyralid, 2,4-D y Picloram, en 3 dosis aplicadas la segunda semana de diciembre, obteniendo como resultado que el ingrediente activo más efectivo fue el Picloram $(0,67$ $-1,33 \mathrm{~kg}$ ia ha-1).

A nivel regional, Salinas (2002) realizó un estudio detallado, el que consistió en evaluar distintos herbicidas del mercado nacional (Cuadro 1).

Cada uno de estos tratamientos fue aplicado por Salinas (2002) en parcelas con dos dosis de nitrógeno $(0 \mathrm{y}$ $60 \mathrm{U} \mathrm{de} \mathrm{N} \mathrm{ha}{ }^{-1}$ ). Salinas (2002), concluye que los herbi- 
Cuadro 1. Tratamientos utilizados en el experimento de Salinas (2002).

Table 1. Treatments used in the experiment by Salinas (2002).

\begin{tabular}{lc}
\hline \multicolumn{1}{c}{ Herbicida } & Dosis i.a ha ${ }^{-1}\left(\mathrm{~g} \mathrm{ha}^{-1}\right)$ \\
\hline 2,4 D & 720 \\
2,4 D+picloram & $720+99,6$ \\
2,4 D+picloram & $720+149,4$ \\
2,4 D+clopyralid & $720+81,8$ \\
2,4 D+clopyralid & $720+122,7$ \\
2,4 D+dicamba & $720+120$ \\
2,4 D & 1440 \\
Picloram+clopyralid & $99,6+81,8$ \\
Picloram+clopyralid & $99,8+122,7$ \\
Picloram+dicamba & $99,6+120$ \\
Picloram & 99,6 \\
Picloram & 149,4 \\
\hline
\end{tabular}

cidas utilizados lograron controlar el crecimiento y desarrollo de $H$. pilosella, independiente de la fertilización nitrogenada. Entre ellos, los más eficaces fueron 2,4-D ( $720 \mathrm{~g} \mathrm{ha}^{-1}$ ) en mezcla con Picloram (99,6 $\mathrm{g} \mathrm{ha}^{-1}$ ó $149,4 \mathrm{~g}$ ha $\left.^{-1}\right)$, o Clopyralid (122,7 $\left.\mathrm{g} \mathrm{ha}^{-1}\right)$ o Dicamba (120 $\left.\mathrm{g} \mathrm{ha}^{-1}\right)$. Además, el 2,4-D (720 $\left.\mathrm{g} \mathrm{ha}^{-1}\right)$ solo o mezclado con Clopyralid $\left(81,8 \mathrm{~g} \mathrm{ha}^{-1}\right)$ lograron controlar la producción de escapos y el crecimiento vegetativo de la especie H. pilosella. La utilización de control químico a través de la aplicación de herbicidas previo a una fertilización disminuye la presencia de $H$. pilosella, favoreciendo a otras especies tales como las gramíneas que son más eficientes en la recuperación del fertilizante, aumentando así la producción de forraje y ocasionando con ella un posible cambio en la composición botánica de la pradera. Para el caso del Pasto ovillo (Dactylis glomerata L.), si bien no hubo diferencias en la producción de biomasa producto de la aplicación de herbicidas si hubo un efecto cuando existió una fertilización de $60 \mathrm{U} \mathrm{ha}^{-1}$ de nitrógeno. Esto se ve reflejado de igual manera en otras gramíneas como Holcus Lanatus L., Vulpia sp y Polygon $s p$, debido a que los herbicidas aplicados son selectivos para hoja ancha las cuales se vieron claramente afectadas. La contribución de la especie T. repens en la pradera se redujo producto de los tratamientos utilizados (herbicidas + nitrógeno), además, el desmedro de su contribución en la pradera, también es debido a que los herbicidas atacan especies de hoja ancha indistintamente y la aplicación de nitrógeno ayuda a gramíneas a mejorar su capacidad de competir por luz, agua y nutrientes (Salinas, 2002).
Finalmente, Salinas (2002) concluye que la fertilización nitrogenada permite una variación en la composición botánica y aumento en la producción total de la pradera, por si sola, pero no logra disminuir la población de H. pilosella. Por lo tanto, se recomienda realizar la aplicación de herbicidas para control de la maleza y aplicaciones de nitrógeno para aumento de producción de gramíneas. Scott y Sutherland, (1993) señalan que T. repens se verá afectado por la aplicación de herbicidas para hoja ancha independientemente de la utilización de fertilizantes, sin embargo, esta especie es la mejor competidora en condiciones edáficas de baja fertilidad.

En la Patagonia no solo existe $H$. pilosella sino que además se encuentran las especies Hieracium praealtum Vill ex Gochnat. (exótica), H. patagonicum (invasora nativa) y Hieracium chilense Less. (nativa). Estas diferentes especies tienen diversos hábitos de crecimiento, destacando a $H$. praealtum la cual tiene una forma más erecta que $H$. pilosella. Para las especies anteriores, los herbicidas 2,4-D, Glifosato, Picloram y Tryclopir siguen siendo los más efectivos en su control (INTA, 2014).

Si bien no se tiene claro cómo ingresó esta planta a la Patagonia se presume que las semillas vinieron mezcladas con otras especies forrajeras (von Moltke, 2009). El primer registro botánico proviene de 1951 en el sector Río de las Minas en la ciudad de Punta Arenas (Domínguez, 2004).

En Nueva Zelanda $H$. pilosella presenta un comportamiento invasor, habiendo reducido un $80 \%$ la producción ganadera en sectores de coironales, al igual que en la región de Magallanes. A consecuencia de lo anterior, ese país, ha investigado y aplicado una modalidad sustentable de control de malezas, que corresponde al control biológico clásico, es decir, a la búsqueda de enemigos naturales en su lugar de origen (Europa) para su introducción en los lugares invadidos (SAG, 2011). La ventaja de este método, en comparación con otros controles de maleza (físico, químico, culturales), son principalmente su bajo costo económico y menor valor ambiental (Grosskopf, 2006).

Grosskopf y Hassler (1998), señalan que el control biológico resultó ser una mejor alternativa que el control químico y mecánico en malezas perennes. Hongos fitopatógenos como Puccinia herracia var. piloselloidarum específico para especies estoloníferas del género Hieracium y Erysipe cichoracearum han sido evaluados en Nueva Zelanda, debido a la capacidad de disminuir la tasa de crecimiento de las malezas entre un 2 y un 12\%. (Gibson y Bosh, 1996).

Entre los insectos controladores destacan: la avispa europea Alaucidea pilosellae y tres polillas del género Oxyptulis. las cuales han sido consideradas como potenciales agentes de control biológico (Scott, 1984; Syrett y Sarospataki, 1993). Wilson et al. (1997) señalan que la avispa Aulacidea pilosellae y la polilla Oxyptu- 
lis subterminalis han sido introducidas a Nueva Zelanda como agentes de control.

En Magallanes se han realizado pruebas de control biológico con Macrolabis pilosellae concluyendo que este insecto es capaz de invadir la planta, cumplir su ciclo y tener la capacidad infectar otras plantas aledañas de $H$. pilosella creando agallas y disminuyendo el potencial biológico del género Hieracium, incluyendo a H. patagonicum, planta que se comporta como maleza, pero es de carácter nativo (SAG, 2011).

\section{CONCLUSIONES}

El género Hieracium aprovecha de manera eficiente la degradación de los suelos magallánicos para su proliferación y de esta manera causar un deterioro en la pradera natural, disminuyendo su diversidad y productividad, afectando la producción ganadera de la región. Se presenta en todo Magallanes, sin embargo, su expansión explosiva ha sido en la zona pampeana de la región. Esto, debido a que este sector presenta condiciones extremas que han permitido la proliferación de $H$. pilosella, como niveles pluviométricos bajos $(<300 \mathrm{~mm}$ año-1) lo que se traduce en una baja capacidad de establecimiento de T. repens y otras especies nativas.

Sin duda la capacidad de proliferación de H. pilosella depende de un gran número de variables, no quedando claro, por ejemplo, si prolifera en suelos ácidos e infértiles o crece en sectores fértiles y acidifica el medio debajo de la planta. Sin embargo, queda claro que el factor que influye en mayor magnitud en la gran expansión de esta planta es el antropogénico.

Hay diferentes métodos de control, aun así, la prevención y rápida erradicación de los manchones es la manera más eficiente y menos costosa. Evitar el sobre pastoreo, la sobre extracción de los nutrientes del suelo y la erosión son las medidas que se deben implementar actualmente en la región.

Se requiere continuar con estudios para evaluar los posibles controles biológicos que puedan atacar la expansión del género Hieracium, sobre todo porque este tipo de controles podría tener efectos secundarios y generar daños en el medio ambiente circundante.

Existen métodos culturales como el pastoreo, fertilización y establecimiento de praderas sembradas; métodos químicos como la aplicación de herbicidas de hoja ancha, y métodos biológicos como hongos fitopatógenos y utilización de insectos, sin embargo, el mejor control es la integración de todos estos métodos y el correcto uso de los recursos naturales. De esta forma, se debe considerar la pronta erradicación de pequeños manchones que aparezcan en los potreros mediante métodos de extracción manual o químicos, evitar el sobrepastoreo y mantener la fertilidad de los suelos.

\section{REFERENCIAS}

Anderson, V.L., 1927. Studies of the vegetation of English chalk. V. The water economy of the chalk flora. Journal of Ecology 15, 72-129.

Beckmann, M., Hock, M., Bruelheide, H., Erfmeier, A., 2012. The role of UV-B radiation in the invasion of Hieracium pilosella - A comparison of German and New Zealand plants. Environmental and Experimental Botany 75, 173-180. https://doi.org/10.1016/j.envexpbot.2011.09.010

Bishop, G.F., Davy, A., 1994. Hieracium pilosella L. (Pilosella officinarum F. Shultz and Shultz-Bip). Journal of Ecology 82(180), 95-210.

Bornkamm, R., 1958. Standortsbedingungen und Wasserhaushalt von Trespen-Halbtrockenrasen (Mesobromion) im oberen Leinegebiet. Flora 156, 23-67.

Boswell, C., Espie, P., 1998. Uptake of moisture and nutrients by Hieracium pilosella and effects on soil in a dry subhumid grassland. New Zealand Journal of Agricultural Research 41, 251-261.

Cipriotti, PA., Rauber, R. A., Collantes, M. B., Braun, K., Escartín, C., 2012. Control measures for a recent invasion of Hieracium pilosella in Southern Patagonian rangelands. Weed Research 52, 98-105. https://doi.org/ doi:10.1111/j.1365-3180.2011.00897.x

Cipriotti, P.A., Collantes, M.B., Escartín, C., Cabeza, S., Rauber, R.B., Braun, K., 2014. Experiencias de largo plazo para el manejo de una hierba invasora de pastizales: El caso de Hieracium pilosella L. en la Estepa Fueguina. Ecología Austral 24, 135-144. http://ojs.ecologiaaustral.com.ar/ index.php/Ecologia_Austral/article/view/16

Clyde, L., 1992. Use of phenoxy herbicides in turfgrass in the United States, in: United States Department of Agriculture National Agricultural Pesticide Impact Assessment Program (Eds)., 1996. Biological and Economic Assessment of Benefits from Use of Phenoxy Herbicides. Special NAPIAP Report. Number 1-PA-96. pp. 1-13. http://www.24d.reviews/PDF/NAPIAP-RIAS-studies/ NAPIAP-1996-Assesment-of-Benefits.pdf

Collantes, M.B., Anchorena, J., Cingolani, A.M., 1999. The steppes of Tierra del Fuego: floristic and growthform patterns controlled by soil fertility and moisture. Plant Ecology 140, 61-75. https://doi. org/10.1023/A:1009727629777

Cossens, G.G., Boswell, C.C., 1993. Residual effects of oversowing, overdrilling and herbicide in the long term control of Hieracium pilosella in Otago, New Zealand. Proceedings of the XVII International Grassland Congress. Massey University, February, 8-21, 1993, Palmerston North, New Zealand, pp. 1730-1733.

Covacevich, N., Uribe, I., Lira, R., 1995. Prospección de pilosella (Hieracium pilosella L.) en terrenos de uso ganadero de la XII Región. Informe. INIA/CRI Kampenaike. SEREMI de Agricultura, Intendencia XII Región, Punta Arenas, Chile. 19 p.

Covacevich, N., Cárdenas, C., 2005. Respuesta al pastoreo de Hieracium pilosella, en: Strauch, O., Covacevich, N., Cárdenas, A. (Eds.), El Manejo sustentable de las praderas naturales de Magallanes. SOCHIPA en Magallanes 30 años. Instituto de Investigaciones Agropecuarias. Actas 
INIA N³5. Punta Arenas, Chile, pp 15-16. http://biblioteca.inia.cl/medios/biblioteca/serieactas/NR33801.pdf

Cruz, G., Lara, A., 1987. Regiones naturales del área de uso agropecuario de la XII región, Magallanes y de la Antártica Chilena. INIA Kampenaike-Intendencia de la XII Región. Punta Arenas, Chile. 20 p.

Díaz-Barradas, C.M., Zunzunegui, M., Álvarez-Cansino, L., Esquivias, M.P., Collantes, M.B., Cipriotti, P.A., 2015. Species-specific effects of the invasive Hieracium pilosella in Magellanic steppe grasslands are driven by nitrogen cycle changes. Plant and Soil 397, 175-197. https://doi. org/10.1007/s11104-015-2608-0

Domínguez, E., 2004. Plantas exóticas presentes en el Parque Nacional Pali Aike, XII Región, Chile. Chloris Chilensis. Revista Chilena de Flora y Vegetación Año 7. № 2 . http://www.chlorischile.cl/paliaikeexo/paliaike.htm

Domínguez, E., Bahamonde, N., Muñoz-Escobar, C., 2011. Plantas invasoras en una turbera de Sphagnum abandonada por la explotación de Turba. Tierra Adentro. Instituto de Investigaciones Agropecuarias (INIA). Punta Arenas, Chile, pp. 78-82. http://www2.inia.cl/medios/ biblioteca/ta/NR38037.pdf

Domínguez, E., 2012. Hieracium pilosella L. ssp. euronotum Nägeli et Peter, Maleza invasora presente en turberas abandonadas y explotadas. Instituto de Investigaciones Agropecuarias. Informativo INIA $N^{\circ} 21$, Centro de Investigación Kampenaike. Punta Arenas, Chile, pp. 1-4. http://www2. inia.cl/medios/kampenaike/noticias/Informativo\%20 21_Hieracium\%20pilosella\%20en\%20Turberas.pdf

Espie, P.R., 1992. The influence of ground cover on hawkweed establishment in fescue tussock grassland, in: Hunter, G.G., Mason, C.R., Robertson, D.M. (Eds.), Vegetation change in tussock grasslands, with emphasis on hawkweeds. New Zealand Ecological Society, Occasional Publication 2, Christchurch, N.Z., pp. 14.

Estrada, J.A., Flory, S.L., 2015. Cogongrass (Imperata cylindrical) invasion in the US: Mechanisms, impacts and threats to biodiversity. Global Ecology Conservation 3, 1-10. https://doi.org/10.1016/j.gecco.2014.10.014

Farman, J.C., Gardiner, B.G., Shanklin, J.T., 1985. Large losses of total ozone in Antarctica reveal seasonal $\mathrm{ClO}_{\mathrm{x}} /$ $\mathrm{NO}_{\mathrm{x}}$ interaction. Nature 315, 207-210. https://doi.org/ doi:10.1038/315207a0

Gibson, R.S., Bosh, O.J.H., 1996. Indicator species for the interpretation of vegetation condition in the St Bathans area, Central Otago, New Zealand. New Zealand Journal of Ecology 20(2), 163-172. http://newzealandecology. org/nzje/1997

Grosskopf, G., Hassler, A., 1998. Investigations on potential biocontrol agents of mouse-ear hawkweed, Hieracium pilosella. Annual Report 1998. CABI-Bioscience Centre, European Station, Switzerland.

Grosskopf, G., 2006. Investigations on three species of Diptera associated with hawkweeds in Europe and their potential for biological control of alien invasive Hieracium spp. in New Zealand and North America. Ph. D. thesis, Christian-Albrechts-Universität, Kiel, Germany, 139 p.

Hernández, L., Marínez-Fernández, J., Cañellas, I., Vásquez de la Cueva, A., 2014. Assessing spatio-temporal rates, patterns and determinants of biological invasions in forest ecosystems. The case of Acacia species in NW Spain.
Forest Ecology and Management 329, 206-213. https:// doi.org/10.1016/j.foreco.2014.05.058

Instituto Nacional de Estadísticas (INE), 2007. VII Censo Agropecuario y Forestal. http://www.ine.cl/estadisticas/censos/censo-agropecuario-y-forestal-2007.

Instituto Nacional de Tecnología Agropecuaria (INTA), 2014. Experiencias de control de Hieracium sp. en Santa Cruz y Tierra del Fuego. Jornadas Ganaderas de Río Gallegos. Río Gallegos, Provincia de Santa Cruz, Argentina.

Knicker, H., Saggar, S., Bäumler, R., McIntosh, P.D., KögelKnabner, I., 2000. Soil organic matter transformation induced by Hieracium pilosella L. in tussock grassland of New Zealand. Biology and Fertility of Soils 32, 194201. https://link.springer.com/content/pdf/10.1007/ s003740000234.pdf

Makepeace, W., 1985a. Some establishment characteristics of mouse-ear and king devil hawkweeds. New Zealand Journal of Botany 23, 91-100.

Makepeace, W., 1985b. Growth, reproduction and production biology of mouse-ear and king devil hawkweed in eastern South Island, New Zealand. New Zealand Journal of Botany 23, 65-78.

Mandal, G., Joshi, S.P., 2014. Invasion establishment and habitat suitability of Chromolaena odorata (L.) King and Robinson over time and space in the western Himalayan forest of India. Journal of Asia-Pacific Biodiversity 7, 391-400. https://doi.org/10.1016/j.japb.2014.09.002

Mcintosh, P.D., Loeseke, M., Bechler, K., 1995. Soil changes under mouse-ear hawkweed (Hieracium pilosella). New Zealand Journal of Ecology 19, 29-34.

Moore, D., 1983. Flora of Tierra del Fuego. Oswestry Anthony Nelson, England, Missouri Botanical Garden, USA.

Pisano, E., 1977. Fitogeografía de Fuego-Patagonia Chilena I.-Comunidades vegetales entre las latitudes 52 y $56^{\circ} \mathrm{S}$. Anales de Instituto de la Patagonia 8(1-2), 122-250.

Rauber, R., Collantes, M.B., Cipriotti, P.A., Anchorena, J., 2013. Biotic and abiotic constraint to a plant invasion in vegetation communities of Tierra del Fuego. Austral Ecology 38, 436-442. https://doi.org/10.1111/j.14429993.2012.02427.x

Rauber, R.B., Cipriotti, P.A., Collantes, M.B., 2014. Local and intermediated-intensity soil disturbances increase the colonization and expansion dynamics of an invasive plant in Southern Patagonian rangelands. Community Ecology 15, 97-93. https://doi.org/10.1556/ComEc.15.2014.1.9

Rauber, R., Cipriotti, P., Collantes, M., Martini, J.P., Frers, E., 2016. Regional Suitability Assessment for the Mouseear Hawkweed (Hieracium pilosella) Invasion in Patagonian Rangelands. Invasive Plant Science and Management 9(4), 242-251. https://doi.org/10.1614/IPSMD-16-00037.1

Rose, A.B., Frampton, C.M., 1999. Effects of microsite characteristics on Hieracium seedling establishment in tall- and short- tussock grasslands, Marlborought, New Zealand. New Zealand Journal of Botany 37(1), 107-118. https://doi.org/10.1080/0028825X.1999.9512617

Saggar, S., McIntosh, P.D., Hedley, C., Knicker, H., 1999. Changes in soil microbial biomass, metabolic quotient, and organic matter turnover under Hieracium (H. pilosella L.). Biology and Fertility of Soils 30, 232-238. https:// doi.org/10.1007/s003740050613 
Salinas, C., 2002. Eficacia de diferentes herbicidas sobre el crecimiento y desarrollo de Hieracium pilosella L. bajo dos niveles de fertilización nitrogenada en una pradera naturalizada de la Región de Magallanes. Tesis Ing. Agr. Universidad Austral de Chile. 96 p.

Scott, D., 1984. Hawkweeds in run country. Review. Tussock grasslands and Mountainlands Institute 42, 33-48.

Scott, D., Robertson, J.S., Archie, W.J., 1990. Plant dynamics of New Zealand tussock grassland infested with Hieracium pilosella. I. Effects of seasonal grazing, fertilizer and overdrilling. Journal of Applied Ecology 27, 224-234. http://www.jstor.org/stable/2403580

Scott, D., Sutherland, B.L., 1993. Interaction between some pastures species and two Hieracium species. New Zealand Journal of Ecology 17(1), 47-51. http://www.jstor. org/stable/24053638

Servicio Agrícola y Ganadero (SAG), 1998. Programa "Prevención, Difusión, Riesgo y Proliferación Hieracium pilosella L. Informe final. Ministerio de Agricultura, Punta Arenas, Chile. 20 p.

Servicio Agrícola y Ganadero (SAG), 2003. El pastizal de Tierra del Fuego. Guía de uso, condición actual y propuesta de seguimiento para determinación de tendencia. Gobierno de Chile. Punta Arenas, XII Región de Magallanes y Antártica Chilena, Chile.

Servicio Agrícola y Ganadero (SAG), 2004a. El pastizal de Magallanes. Guía de uso, condición actual y propuesta de seguimiento para determinación de tendencia. Gobierno de Chile. Punta Arenas, XII Región de Magallanes y Antártica Chilena, Chile.

Servicio Agrícola y Ganadero (SAG), 2004b. El pastizal de Ul- tima Esperanza y Navarino. Guía de uso, condición actual y propuesta de seguimiento para determinación de tendencia. Gobierno de Chile. Punta Arenas, XII Región de Magallanes y Antártica Chilena, Chile.

Servicio Agrícola y Ganadero (SAG), 2011. Programa de control biológico de Hieracium pilosella L. (Compositae: Asteraceae) en la Región de Magallanes y Antártica Chilena. Informe Final. Punta Arenas, Chile.

Syrett, P., Sarospataki, M., 1993. Prospects for biological control of hawkweeds with insects. Proceedings of the $6^{\text {th }}$ Australasian Grasslands Invertebrate Ecology Conference, AgResearch, Hamilton, New Zealand, pp. 426-432.

Steer, M.A., Norton, D.A., 2013. Factors influencing abundance of invasive hawkweeds, Hieracium species, in tall tussock grasslands in the Canterbury high country. New Zealand Journal of Botany 51(1), 61-70. https://doi.or $\mathrm{g} / 10.1080 / 0028825 X .2012 .753096$

Strauch, 0., 2012. Medidas de Mitigación y Control de la Pilosella: Una primera aproximación. Instituto de Investigaciones Agropecuarias, Informativo INIA N ${ }^{\circ} 27$. Centro de Investigación Kampenaike, Punta Arenas, Chile. pp. 1-3. https://asociacionruraltdf.files.wordpress. com/2012/06/medidas-de-mitigacic3b3n-y-controlde-pilosela-una-primera-aproximacic3b3n.pdf

von Moltke, C., 2009. Distribución Hieracium pilosella L. en la provincia de Magallanes. Tesis. Escuela de Ciencias y Tecnología en Recursos Agrícolas y Acuícolas. Universidad de Magallanes, Punta Arenas, Chile. 50 p.

Wilson, L., McCaffrey, J.P., Quimb, P.C. Birdsall, J.L., 1997. Hawkweeds in the Northwestern United States. Rangelands 19(4), 18-23. 